\title{
Sarcopenia: A Geriatric Giant Facing a Huge Transition
}

\author{
Sang Yoon Lee \\ Department of Rehabilitation Medicine, Seoul National University College of Medicine, SMG-SNU Boramae Medical Center, Seoul, Korea
}

Since 2010, when the European Working Group on Sarcopenia in Older People (EWGSOP) first presented guidelines for the diagnosis of sarcopenia, ${ }^{1)}$ worldwide medical interest in this condition has exploded. The International Classification of Diseases, 10th Revision, Clinical Modification (ICD-10-CM) code for sarcopenia (M62.84) represents a major step forward in recognizing sarcopenia as a disease ${ }^{2)}$ and has been registered in the disease code systems of countries worldwide. The number of papers related to sarcopenia indexed in the PubMed database has increased exponentially, with more than 2,000 papers published per year in the last 2 years (Fig. 1). Thus, sarcopenia has become an important giant in geriatric medicine. ${ }^{3)}$

This geriatric giant has undergone a huge transition in the past year with the announcement by the EWGSOP of a revised consensus on sarcopenia 9 years after the first consensus. ${ }^{4)}$ This update maintains the three key axes for sarcopenia diagnosis; namely, muscle mass, muscle strength, and physical performance. However, unlike previous algorithms that measured walking speed first, muscle strength was first measured through grip strength or the chair stand test, which can be easily applied in the clinic to identify "probable sarcopenia." In cases of confirmed sarcopenia, the algorithm can be used to diagnose "severe sarcopenia" based on physical performance. In addition, a step was added to identify cases in the clinic through the application of the SARC-F questionnaire (The SARC-F questionnaire is composed of five domains: Strength, Assistance with walking, Rise from a chair, Climb stairs and Falls). The cut-off values for muscle mass, muscle strength, and physical performance were updated based on the clinical data accumulated to date. In line with this, the Asian Working Group for Sarcopenia (AWGS) also updated the consensus report in 2019, , to include "case finding" in the diagnostic algorithm consistent with that in the revised EWGSOP. Calf circumference and the SARC-F were also added. In addition, while the revised EWGSOP regarded the five-time chair stand test as a diagnostic method for "muscle strength," the AWGS 2019 included this test in "physi- cal performance" based on the 6-Meter Walk Test and Short Physical Performance Battery. The development and evolution of efforts to more easily and accurately diagnose and classify sarcopenia in clinical practice are anticipated.

The first-line treatment for sarcopenia is exercise and nutritional intervention. Strong evidence supports the application of progressive resistance exercises suitable for older adults as well as targeted protein intake and provision of nutrition. ${ }^{6,7)}$ However, more reliable and sustainable interventions in geriatric medicine additionally require pharmacological interventions for sarcopenia. ${ }^{8)}$ Clinical trials of new drugs targeting multiple molecules are ongoing. The positive results of trials of myostatin inhibitors, selective androgen receptor modulators, and vitamin $\mathrm{D}$ targeting various sarcopenia phenotypes have been published. For instance, bimagrumab, a monoclonal antibody of activin receptor type $2 \mathrm{~B}$, has been reported in clinical trials targeting diseases such as inclusion body myositis $^{9)}$ and chronic obstructive pulmonary disease. ${ }^{10)}$ Finally, the results of an international multicenter clinical trial (38 sites in 13 countries) on the treatment of sarcopenia in community-dwelling older adults were finally released in 2020. ${ }^{11)}$ While the sarcopenia drug treatments evaluated in clinical trials to date have the effect of increasing muscle mass, this increased mass did not lead to improved muscle strength or physical performance. But in the near future, science certainly expects to find the right answer.

In 2020, we faced the coronavirus disease 2020 pandemic, an unprecedented global infectious disease that continues to this day. As of February 2021, 113 million people worldwide have been confirmed to have COVID-19, with a mortality rate of approximately $2.22 \%$. Older patients with COVID-19 complain of severe muscle weakness and poor physical function even after recovering from acute illness. ${ }^{12,13)}$ In addition, strong social distancing and lockdowns implemented in almost all countries will further accelerate frailty in this population. Therefore, the phenomena we have experienced over the past year due to the pandemic have further increased the prevalence of sarcopenia in older adults; thus, clini- 


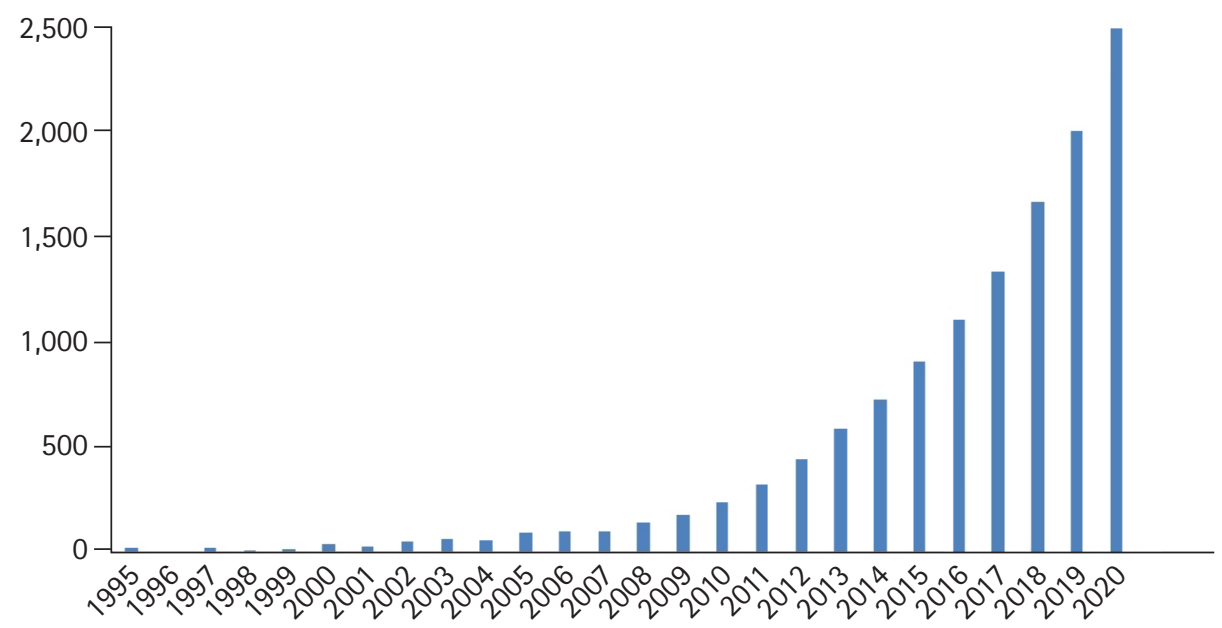

Fig. 1. Annual numbers of articles on sarcopenia indexed in the PubMed database (search query: sarcopenia).

cians should prepare for a new phase after COVID-19.

Sarcopenia, which has become a geriatric giant, is already growing in size and has become a protagonist requiring our attention in a super-aged society. This giant stands amid the huge inflection of revised diagnoses, new treatments, and COVID-19. If we do not tame this giant in advance, it will present as a bigger and more terrifying challenge to the next generation.

\section{ACKNOWLEDGMENTS}

\section{CONFLICT OF INTEREST}

The researcher claims no conflicts of interest.

\section{FUNDING}

This work was supported by the National Research Foundation of Korea (NRF) grant funded by the Korea government (MSIT) (No. 2019R1C1C100632).

\section{REFERENCES}

1. Cruz-Jentoft AJ, Baeyens JP, Bauer JM, Boirie Y, Cederholm T, Landi F, et al. Sarcopenia: European consensus on definition and diagnosis: report of the European Working Group on Sarcopenia in Older People. Age Ageing 2010;39:412-23.

2. Anker SD, Morley JE, von Haehling S. Welcome to the ICD-10 code for sarcopenia. J Cachexia Sarcopenia Muscle 2016;7:5124.

3. Sayer AA. Sarcopenia the new geriatric giant: time to translate research findings into clinical practice. Age Ageing 2014;43:7367.

4. Cruz-Jentoft AJ, Bahat G, Bauer J, Boirie Y, Bruyere O, Cederholm T, et al. Sarcopenia: revised European consensus on defini- tion and diagnosis. Age Ageing 2019;48:16-31.

5. Chen LK, Woo J, Assantachai P, Auyeung TW, Chou MY, Iijima K, et al. Asian Working Group for Sarcopenia: 2019 Consensus Update on Sarcopenia Diagnosis and Treatment. J Am Med Dir Assoc 2020;21:300-307. e2.

6. Vlietstra L, Hendrickx W, Waters DL. Exercise interventions in healthy older adults with sarcopenia: A systematic review and meta-analysis. Australas J Ageing 2018;37:169-83.

7. Yoshimura Y, Wakabayashi H, Yamada M, Kim H, Harada A, Arai H. Interventions for treating sarcopenia: a systematic review and meta-analysis of randomized controlled studies. J Am Med Dir Assoc 2017;18:553.e1-553.e16.

8. Kwak JY, Kwon KS. Pharmacological interventions for treatment of sarcopenia: current status of drug development for sarcopenia. Ann Geriatr Med Res 2019;23:98-104.

9. Sivakumar K, Cochrane TI, Sloth B, Ashar H, Laurent D, Tanko LB, et al. Long-term safety and tolerability of bimagrumab (BYM338) in sporadic inclusion body myositis. Neurology 2020;95:e1971-e1978.

10. Polkey MI, Praestgaard J, Berwick A, Franssen FM, Singh D, Steiner MC, et al. Activin type II receptor blockade for treatment of muscle depletion in chronic obstructive pulmonary disease: a randomized trial. Am J Respir Crit Care Med 2019;199:313-20.

11. Rooks D, Swan T, Goswami B, Filosa LA, Bunte O, Panchaud N, et al. Bimagrumab vs optimized standard of care for treatment of sarcopenia in community-dwelling older adults: a randomized clinical trial. JAMA Netw Open 2020;3:e2020836.

12. Jang SN, Kim CO. Care inequality among older adults during the COVID-19 pandemic. Ann Geriatr Med Res 2020;24:229-31.

13. Morley JE. Editorial: Sarcopenia: 2020. J Nutr Health Aging $2021 ; 25: 278-80$. 
Corresponding Author: Sang Yoon Lee, $\mathrm{MD}, \mathrm{PhD}$

Department of Rehabilitation Medicine, Seoul National University College of Medicine, SMG-SNU Boramae Medical Center, 20 Boramae-ro 5-gil,

Dongjak-gu, Seoul 07061, Korea

E-mail:1sy126@snu.ac.kr

ORCID: https://orcid.org/0000-0002-2906-3094

Received: February 26, 2021; Revised March 8, 2021; Accepted March 9, 2021 